equilibrium is the resultant of normal customary external impressions, and the symptoms of agoraphobia are produced by the absence or clashing of one or more of these impressions." This explanation seems to me hardly satisfactory, as my patient insists very strongly that there is a distinct difference between the giddiness associated with his bilious attacks and the giddiness which accompanies his agoraphobic attacks; again, companionship can hardly affect a man's appreciation of vertical lines, by which Dr. Stewart thinks the patients guide themselves, and also we find that the mental ideas of infinity, vastness, \&c., are just as potent to produce the attacks as are external im pressions.

To me the case seems essentially a form of mental depres. sion in certain faculties unassociated with delusion and closely allied to what is called simple melancholia. There are disorders of perception; bright light, loud noises, likes and dislikes to persons and things are more pronounced and uncalled for, there is increased irritability to all external impressions, and these are accompanied with some form of deteriorated physical health; but I think the seat of this condition is certainly central, not peripheral. May it not be due to some functional irritation of the cerebral cortical sensory centres, which Ferrier calls the anatomical substrata of ideation?

The prognosis in this case is good, and he has always improved and recovered from his nervousness with the recovery of his usual good general health.

Treatment has been with the object of improving the general health by small doses of cod-liver oil, attention to diet, and the avoidance of such associations as aggravate the disorder. Great relief has been found from wearing a pair of dark-purple spectacles, especially upon bright days. The recumbent position, bathing the head with cold water, or a small dose of some stimulant, will often ward off an attack for some time. The peculiar pain in the back of the head is best relieved by pressure of the hands. A dose of bromide of potassium and strychnia will enable him to mount a hill or do some work which he could not have accomplished and would have shrunk from without it, and he finds he requires a quarter of an hour before the medicine acts. Other medicine he takes from which he is confident he receives benefitnamely, nux vomica, iron, and hydrobromic acid.

Wigan.

\section{THE SURGICAL TREATMENT OF DEBILITY, ASIATIC CHOLERA, ETO.}

\section{BY J. R. UHLER, M.D.}

A FEW years ago, having a patient with stricture of the csophagus under my care, whom I was unsuccessfully trying to nourish by the rectum, I determined to take advantage of some of the little pores in the subcutaneous tissue to introduce more liquid into the system. For this purpose a nasal douche, with a two-way cock attached to soft rubber tubes, was employed, and at the end of each tube, where the nozzle is usually placed, the cylinder of a hypodermic syringe, deprived of its piston but retaining the needle, was substituted, making a reservoir apparatus somewhat like an aspirator, with two tubes and no valves. This receptacle was filled with whisky and water and other thin nutritive fluids, and the needles were thrust deeply into the areolar tissue of the extremities, while the reservoir was raised slightly above the level of the body to prevent the entrance of air. The fluid was prevented from escaping by pinchcocks close at the ends of the tubes, but at the moment of insertion a few drops were allowed to flow from the needles to show that it was right, and the pincheocks were then removed. The reservoir was now raised high enough to permit the liquid to pass readily through the needles by natural flow, giving a constant, gradual supply to the tissues, care being taken not to elevate it too much, so as to avoid undue pressure or large collections of fluid under the skin, the object being only to supply the fluid a little faster than it can be absorbed, and to avoid abscesses. This device worked very well on the first day, affording much relief to the patient; but in the course of time the needles became loose, and largersized ones had to be substituted. Since then I have used it a few times as a temperary expedient in the vomiting of pregnancy, coma, phthisis, emaciation, after great loss of blood, and when food could not be swallowed, retained, digested, or absorbed. I have also thought (and find from the Journal of the A merican Medical Ass jciation of August 9th, 1884, that Dr. Todd has suggested the same treatment in a different way) that sterilised artificial serum, composed of the chlorides of sodium and potassium in smal quantity, dissolved in distilled water, can thus be supplied to the depleted system in cholera from one or more re servoirs connected with three or four tubes and aspiration needles inserted into the extremities. A better plan, however, for cholera, and one which acts locally, and can be combined with this when necessary, is to fill the peritoneal sac, without distension, with sterilised artificial serum, injected at the temperature of the body by the aspirator or other appropriate instruments. The fluid is thus brought in contact with the other side of the diseased intestines, where it can be withdrawn as wanted, and will, like ascitic fluid, act by pressure, diminishing somewhat the size of the bloodvessels and lumen of the bowels; and, as it is exosmosed, washing away or diluting those low forms of life that are said to be the cause of the disease. The fluid when absorbed will also prevent that tarry condition of the blood which stops circulation, and will not interfere except by dilution with opium, acids, gases, or any other antiseptic remedies that may be thought proper to be applied to the interior of the stomach and bowels. For security, the peritoneum may be cut down upon with antiseptic precautions by an ordinary scalpel, and when it is reached a minute hole be made by pinching up the part and incising it so as to admit the dome-shaped aspirator needle or other purified tube. The mouth of the reservoir or aspirator bottle should be closed by sterilised cotton-wadding, and the wound be antiseptically dressed immediately after the operation. 'The proper time to fill the peritoneal sac is when the serum is rapidly draining off from the bowels, and before collapse occurs, but I would not hesitate to use it in that condition so long as there seemed to be any prospect of absorption. Baltimore, Margland, U.S.A.

\section{EXCISION OF LOWER JAW FOR RECUR- RENT MYELOID EPULIS.}

\section{BY W. ALEX. MACKAY, M.B., C.M. EdIN.}

EMILIA DE G—, aged twenty-six, born in Madrid, had the last lower molar tooth extracted in 187\%. The extraction was followed by a good deal of bleeding and pain. Three months after she felt a hard painless swelling on the outer aspect of the bone corresponding to the situation of the extracted tooth. This slowly increased till in 1881 it had attained the size of an orange. The patient then consulted Senor Don Enrique Perez Andres, who removed the tumour. In three months the growth had returned in the same place, hard and painless as before, but growing now more rapidly, so that on December $20 \mathrm{~s}, 1883$, the left cheek was found tightly stretched over a tumour which entirely surrounded the left half of the lower jaw. The growth extended upward to the articulation, inward half way across the mouth, forward it reached the symphysis, and down. wards it projected on the neck. The alveolar border of the upper jaw was carried inwards nearly to the middle line, the teeth of this jaw being received into a deep sulcus on the upper surface of the tumour. Examination of that part accessible from the mouth gave rise to pain, and this part also bled when touched, and was of a bright, fleshy, red colour. The cheek, which was adherent, was so iightly stretched that it had ulcerated at the most prominent point but the ulcer had healed. Neighbouring glands were unaffected. The patient was of a nervous temperament, with a fast and weak pulse; very aniemic and emaciated, as she could not masticate her food, and bad lived for some months entirely on fluids. There was slight congestion at the apex of the right lung. Heart and other organs were healthy. The urine was normal in quantity, and did not contain albumen. Her family history was good, and embraced no instance of tumour.

On Dec. $26 \mathrm{th}, 1883$, the patient being under an anæesthetic, the following operation was performed. Entering the knife at the temporo-maxillary articulation, an incision was carried down along the posterior border of the ascending ramus, round the angle, and along the body of the jaw for 